OPEN ACCESS

Edited by:

Benyi $L i$,

University of Kansas Medical Center,

United States

Reviewed by:

Carmela Spagnuolo,

Italian National Research Council, Italy

Ruibao Chen,

Huazhong University of Science and

Technology, China

*Correspondence:

Guojun Wu

wuguojun@csu.edu.cn

Specialty section:

This article was submitted to

Pharmacology of

Anti-Cancer Drugs,

a section of the journal

Frontiers in Oncology

Received: 08 August 2020 Accepted: 04 November 2020

Published: 04 December 2020

Citation:

Wu Y, Xu J, Liu Y, Zeng Y and Wu G

(2020) A Review on Anti-Tumor

Mechanisms of Coumarins.

Front. Oncol. 10:592853.

doi: 10.3389/fonc.2020.592853

\section{A Review on Anti-Tumor Mechanisms of Coumarins}

\author{
Yi Wu ${ }^{1}$, Jing $X u^{1}$, Yiting Liu ${ }^{1}$, Yiyu Zeng ${ }^{1}$ and Guojun $W u^{2 *}$ \\ ${ }^{1}$ School of Stomatology, Central South University, Changsha, China, ${ }^{2}$ Department of Microbiology, School of Basic Medical \\ Science, Central South University, Changsha, China
}

Coumarins are a class of compound with benzopyrone as their basic structure. Due to abundant sources, easy synthesis, and various pharmacological activities, coumarins have attracted extensive attention from researchers. In particular, coumarins have very significant anti-tumor abilities and a variety of anti-tumor mechanisms, including inhibition of carbonic anhydrase, targeting PI3K/Akt/mTOR signaling pathways, inducing cell apoptosis protein activation, inhibition of tumor multidrug resistance, inhibition of microtubule polymerization, regulating the reactive oxygen species, and inhibition of tumor angiogenesis, etc. This review focuses on the mechanisms and the research progress of coumarins against cancers in recent years.

Keywords: anticancer agents, carbonic anhydrase inhibitors, caspase-dependent apoptosis, coumarin, reactive oxygen species

\section{INTRODUCTION}

Cancer is the second leading cause of death worldwide after cardiovascular disease, and a major threat to human health. Coumarins are a class of natural compounds widely found in a variety of plant families, including Umbelliferae, Compositae, Leguminosae, Rutaceae, mulberry, mignonette, and thyme (1). They have a wide range of pharmacological activities, such as anti-inflammatory, anticoagulant, antibacterial, antifungal, antiviral, anticancer, anti-hypertensive, etc (2). Moreover, coumarin is characterized by a simple structure, benzopyrone, on which there are multiple substitution sites. According to the different substituents, coumarins can be divided into five classes: simple coumarins, pyranocoumarins, furocoumarins, dicoumarin, and isocoumarin (Figure 1). By modifying the structure of coumarin and introducing functional groups, researchers have synthesized more complex and diverse coumarin derivatives with more application value and more performance. In recent years, coumarin and its derivatives have shown an extremely wide and inestimable potential in the field of anti-tumor therapy. Their antitumor mechanisms are very diverse, including inhibiting carbonic anhydrase (CA), targeting PI3K/ Akt/mTOR signaling pathway, inhibiting multiple drug resistance (MDR), and inducing apoptosis, etc. In this review, the anti-tumor mechanisms and the research progress of coumarins have been emphasized in recent years.

\footnotetext{
Abbreviations: CA, Carbonic anhydrase; MDR, Multiple drug resistence; HIF, Hypoxia-inducible transcription factor; TCHD, The total coumarins of Hedyotis diffusa; PARP, Poly-ADP-ribose polymerase; Bcl-2, B cell lymphoma-2; P-gp, P-glycoprotein; ROS, Reactive oxygen species; NQO1, NAD(P)H quinone oxidoreductase-1; ALDH, Aldehyde dehydrogenase; TCHs, Triphenylethylene-coumarin hybrids; STAT3, Signal transducer and activator of transcription-3; MCT, Monocarboxylate transporter; AKR1B10, Aldo-Keto Reductase 1B10.
} 


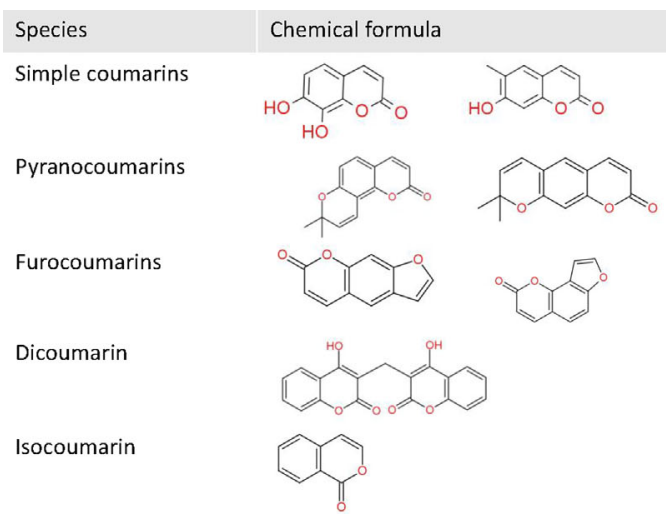

Introduction

Only have substituents on the benzene

ring of coumarin

The C- 6 / C- 8 on the benzene ring is

replaced by the pyran group

The c-6/ C- 8 on the benzene ring is

replaced by the furan group

The dimer of coumarins

The isomer of coumarin

FIGURE 1 | The basic classification of coumarins. This figure shows some chemical formulas and basic introduction of five classes coumarins.

\section{ANTICANCER MECHANISMS}

\section{Coumarins Act as CA Inhibitors}

$\mathrm{CA}$ is a zinc-containing metal enzyme, catalyzing reversible hydration of carbon dioxide, maintaining $\mathrm{pH}$ inside and outside the cell, and transmembrane transport of ions, etc., and affecting a variety of metabolic activities (3). There are 16 different $C A$ subtypes at least, among which CA I and CA II belong to a cytoplasmic enzyme and CA IX and CA XII belong to transmembrane protease. Tumor cells and their microenvironment are often hypoxic and increased glycolysis to meet increased metabolic needs, leading to a large accumulation of lactic acid. The expression of CA IX and CA XII in tumor cells is strongly induced by hypoxia-inducible transcription factor (HIF). The two types of carbonic anhydrase can catalyze the reversible hydration of carbon dioxide, convert it into bicarbonate ions and hydrogen ions, and expel hydrogen ions out of the cells to make the tumor cells maintain a slightly intracellular alkalinity and an extracellular acidity, which is conducive to the growth and spread of primary tumors and the development of drug resistance, leads to the formation of metastases $(3,4)$. CA IX and CA XII are overexpressed in various malignant tumors and can be used as important targets for the design of anticancer compounds.

Based on the structure of Coumarin and sulfonamide, Belma (5) designed a series of compounds and tested their abilities to inhibit the expression of CA I, CA II, CA IX, and CA XII in colon cancer cells. They found that these compounds can effectively restrain the CA IX and CA XII expression. 4-(((2-((1-(3-((2-oxo-2h-chromen7-yl)foxy)propyl) 1h-1,2,3-triazol-4-yl)methoxy)naphthalen-1-yl)methylene)amino) methyl) benzenesulfonamide (8i) exhibited the ability of selective inhibition of cell proliferation via specific inhibition of CA IX and CA XII expression in human colon cancer cells with the $\mathrm{Ki}$ is $45.5 \mathrm{nM}$ and $596.6 \mathrm{nM}$, respectively. Belma (6) also synthesized 16 kinds of bis-coumarin derivatives (5a$5 p)$ containing three thiazole rings and alkyl chains, and assessed their inhibitory activities against CA I, CA II, CA IX, and CA XII. They found that all synthetic compounds showed selective inhibitory activities against tumor-related CA IX and CA XII in the high nM range. Among them, 4-methyl-7-((1-(12-(2h-(2-oxo-chromen-7-yl) foxy)dodecyl) 1h-1,2,3-triazol-4-yl)methoxy)-2h-chromen-2-one (5p) and 4-methyl-7-((1-(1-(2h-(2-oxo-chromen-7-yl) foxy) decyl) 1h- 1,2,3-triazol-4-yl)methoxy)-2h-chromen-2-one (5n) showed the highest CA XII and CA IX inhibition with the Ki is 144.6 $71.5 \mathrm{nM}$, respectively.

Some 1-aryl and 2-aryl-substituted Coumarin derivatives showed selective inhibitory activities to CA IX and CA XII (7). Angapelly (8) synthesized a series of 4-sulfamoylphenyl/ sulfocoumarin benzamides and evaluated their inhibitory effects on five subtypes of CA. They found that all these sulfocoumarin compounds are weak or invalid inhibitors for CA I and CA II, but can effectively inhibit tumor-related CA IX and CA XII in the high nanomolar to micromolar ranges.

$\mathrm{Yu}$ (9) synthesized a series of new dihydroartemisinincoumarin hybrids. These hybrids showed moderate cytotoxicity to human colon and breast cancer cells. The mechanism included targeting CA IX, resulting in the intracellular accumulation of hydrogen ions, arresting the cell cycle at G0/G1 phase, and a sharp decline in mitochondrial membrane potential, promoting apoptosis and inducing ferroptosis.

In addition, Ferraroni (10) synthesized a series of Coumarins and corresponding 2-thioxocoumarines and tested their inhibitory activities to human carbonic anhydrase CA I, CA II, CA IX, and CA XII, and found that 7-[(1-Phenyl-1H-1,2,3-triazol-4-yl) methoxy]-2H-chromen-2-one (15) and 7-[(1-phenyl-1H-1,2,3triazol-4-yl)methoxy]-2H-chromene-2-thione (16) was the most effective and the selective inhibitors of CA IX and CA XII, with the Kis are comparable to or slightly lower than the standard sulfonamide AAZ. To explore the inhibitory mechanism of 2thioxocoumarin to CA, Ferraroni (10) examined the X-ray crystal structure of 6-hydroxy-2-thioxocoumarin which bound to CA II and found that the inhibitory mechanism of 2-thioxocoumarin is quite different from other coumarins, which work as zinc binders or occlusion of the active site entrance, anchor to the metal-ioncoordinated water/hydroxide ion, or bind to outside of the active sites, then hydrolyzed by CA, finally form the corresponding 2hydroxycinnamic acid derivatives. However, the X-ray crystal structure of CA II- thioxocoumarin adducts showed that the exosulfur atom is anchored to the zinc-coordinated water molecule, 
whereas the scaffold establishing favorable contacts with the amino acid residues from the active sites.

\section{Coumarins Can Inhibit PI3K/AKT/mTOR Signaling Pathway}

PI3K is an intracellular phosphatidylinositol kinase. PI3K can activate a series of protein kinase (PKA, PKC, and PKB) and plays an important role in the cell proliferation, apoptosis, migration, and differentiation. AKT, a serine/threonine kinase, also known as $\mathrm{PKB}$, is one of the key downstream target sites for $\mathrm{PI} 3 \mathrm{~K}$, and it is closely related to the cell proliferation and apoptosis. AKT can activate $\mathrm{CDK} 4$ and $\mathrm{CDK} 2$, regulate cycle-dependent protein inhibitor P27, and enable cells to complete the cell cycle successfully. AKT can also show its antiapoptotic effect through various channels, such as inactivating Bax and caspases family, inhibiting the activity of GSK3, which can accelerate cell apoptosis by degrading cytoskeleton protein $\beta$-catenin and inhibiting cell adhesion, activating transcription factor NF- $\mathrm{\kappa B}$ and promoting the repair of cellular DNA damage, inhibiting the expression of proapoptotic gene FasL, inhibiting the release of cytochrome $\mathrm{C}$ and apoptotic factors from mitochondria, and so on. mTOR is also a serine/threonine kinase and is a downstream target site for AKT. Activated mTOR can activate translation inhibition molecule (4E$\mathrm{BP} 1$ ) and ribosomal protein p70S6K. When $4 \mathrm{E}-\mathrm{BP} 1$ is phosphorylated, its binding ability to eIF-4E is weakened, and eIF-4E binds to other translation initiation factors to initiate protein translation. Activated p70S6K can also promote protein synthesis. PI3K/AKT/mTOR is a very important signaling pathway in cell survival, growth, metabolism, proliferation, differentiation, metastasis, and cell cycle regulation $(11,12)$. The signaling pathway is closely related to the pathogenesis of tumors because of often mutation in human tumor cells. Abnormal activation of this pathway plays an important role in tumor development, progression, and drug resistance, thus this pathway is an important target for tumor therapy $(13,14)$.

Wang (15) synthesized 15 kinds of benzylsulfone coumarin derivatives (5a-5o) and found that 3-[(4-fluorobenzyl)sulfonyl]-6nitro-2h-chromen-2-one (5h) and 6-bromo-3-[(4-fluorobenzyl) sulfonyl] -2h-chromen-2-one (5m) exibited the strongest inhibitory activities against PI3K, with inhibition rates of $50.3 \%$ and $50.8 \%$ at $20 \mu \mathrm{M}$ respectively in vitro experiments. In addition, $5 \mathrm{~h}$ and $5 \mathrm{~m}$ had broad-spectrum anti-tumor activities, with the IC50 values for 5 tumor cell lines (Hela, HepG2, H1299, hct-116, and McF-7) ranging from (18.1-32.6) $\mu \mathrm{M}$ and (29.3-42.1) $\mu \mathrm{M}$, respectively. Moreover, $5 \mathrm{~h}$ and $5 \mathrm{~m}$ can appropriately embedded into the active sites of $\mathrm{PI} 3 \mathrm{~K} \alpha$ and $\mathrm{PI} 3 \mathrm{~K} \beta$, and interact with important residues.

5-methoxypsoralen, also known as bergamot and parsley alkali, is a natural product of furocoumarins and usually isolated from psoralen. Guo (16) found that 5-methoxypsoralen inhibits the expression and phosphorylation of PI3K, Akt, and mTOR in human glioma cells, thereby completely down-regulating the expression and activation of the PI3K/Akt/mTOR signaling pathway. 5-methoxypsoralen can also cause DNA damage by fragment the DNA of human glioma cells, and induce the appearance of autophagy vacuoles.
Isofraxidin is a natural Coumarin compound. Shen (17) found that isofraxidin can inhibit the phosphorylation of Akt in human colorectal cancer cells.

Hedyotis diffusa is an ethnic medicine used for anti-cancer treatment in Chinese medicine clinics. The total coumarins of Hedyotis diffusa (TCHD) is a kind of extract with antiproliferative activity. Jiang (18) tested the apoptosis inductive effect of TCHD on human myelodysplastic syndromes cell line (SKM-1) and found that TCHD could inhibit the expression of PI3K, Akt and the phosphorylation of Akt and P65, thereby inducing NF- $\mathrm{KB}$ inactivation.

Imperatorin show anti-tumor properties by inhibiting the phosphorylation of PI3K, Akt, and mTOR in human gastric adenocarcinoma cells (19). Dong (20) synthesized a novel hybrid of 3-benzyl coumarin deca-B-ring derivative and nitric oxide donor benzenesulfonyl furan glycans, and confirmed that this hybrid could inhibit the expression of mTOR and the phosphorylation of mTOR and Akt in non-small-cell lung cancer cells.

Dahong (21) isolated Ferulin C, a natural sesquiterpene coumarin, from Ferula ferulaeoides, and tested its anti-proliferative activity against breast cancer cells in vitro and in vivo. In vitro, by decreasing the expression and nuclear localization of p-AKTSer473, Ferulin C $(20 \mu \mathrm{M})$ significantly inhibited the expression and phosphorylation of AKT and its downstream member mTOR, thus inducing autophagy of tumor cells.

\section{Coumarins Can Affect Apoptotic Associated Proteins}

Caspase-2, -3, -6, -7, -8, -9, and -10 are the key mediators of apoptosis in caspases family. Caspase-2, $-8,-9$, and -10 are the initiators of apoptosis. After receiving the apoptosis signal, caspase2 can be self-catalyzed to activate itself, and initiate apoptosis. Caspase- $3,-6$, and -7 are executers of apoptosis, activated by upstream promoters, can hydrolyze many of the structural and functional proteins, such as poly-ADP-ribose polymerase (PARP) (22). PARP is an important DNA repair enzyme activated by the damaged DNA, identify and bind to the damaged DNA and poly ADP glycosylated DNA repair-related proteins and get involved in the DNA repair process, maintaining the survival and stability of cells. However, when DNA is severely damaged, over-activated PARP will lead to cell death because of the energy exhaustion in the repair process. In addition, poly(ADP-ribose) generated by PARP activation can destroy mitochondria and release the apoptosisinducing factor. During apoptosis, caspase- 3 is activated and PARP is lysed to avoid unnecessary repair of dying cells. Therefore, lysed PARP is considered as an important indicator of caspase-3 activation (23). B cell lymphoma-2 (Bcl-2) is one of the most important oncogenes in apoptosis research. The Bcl-2 family includes pro-apoptotic and anti-apoptotic proteins. Bad, Bax, and PUMA are pro-apoptotic proteins in the Bcl-2 family, which are mainly located in the cytoplasm. Once induced by apoptotic factors, they converge to the outer membrane of mitochondria to form transmembrane channels, which enable mitochondria to release cytochrom $\mathrm{C}$, and activate caspases, thereby promoting apoptosis. $\mathrm{Bcl}-2$ is an anti-apoptotic protein in the Bcl-2 family, which is mainly located on the outer membrane of mitochondria and 
inhibits cell apoptosis by blocking the release of cytochrome C (22). Coumarin compounds can regulate the expression of these apoptosis-related proteins and achieve the goal of tumor inhibition.

Ferulin C, a natural sesquiterpene coumarin, is extracted from from Ferula ferulaeoides. In breast cancer cells, Ferulin C (20 $\mu \mathrm{M})$ substantially elevated the expression of Bax, and reduced the expression of Bcl-2. Ferulin $\mathrm{C}$ also caused the cleavage of caspase3, caspase7, caspase9, and PARP, which indicated the activation of the classical mitochondria apoptotic pathway (21).

Yao (24) extracted a series of coumarin compounds from Juglans mandshurica with $75 \%$ ethanol and tested the cytotoxicity of these compounds to two kinds of hepatocellular carcinoma cell lines in vitro. The experiments showed that some compounds had moderate anti-tumor activities to these cell lines. The results of Western blot analysis showed that under the action of xanthyoxylin (compound 2) and 6, 7, 8-trimethoxyl-coumarin (compound 5), the levels of lysed caspase-7 and PARP increased, and the levels of pro-caspase-7 decreased.

When combined with Akt inhibitor MK2206, isofraxidin had a synergistic effect on liver cancer cells, significantly decreasing the expression of anti-apoptotic protein Bcl-2 and increasing the expression of pro-apoptotic proteins, including caspase-3, caspase- 9 and Bax (17). In addition, caspase-3, -8, -9, and PARP in SKM-1 were significantly activated by TCHD. The single-stranded DNA bind to the overacted PARP and break it to poly(ADP-ribose), which destroys mitochondria and releases apoptosis-inducing factor (18). Elshemy (25) synthesized three series of coumarin hybrids by hybridizing 8-methoxy coumarin with three bioactive moieties, chalcone $2 \mathrm{a}-\mathrm{c}$, acrylohydrazide $4 \mathrm{a}-$ $c$, and pyridine $6 \mathrm{a}-\mathrm{b}$ and $7 \mathrm{a}-\mathrm{b}$. These coumadin hybrids can upregulate the expression of caspase- 3 proteins in HCC cell lines and caspase- 3 and caspase- 9 proteins in leukemia cell lines, and down-regulate the expression of $\mathrm{Bcl}-2$ and the up-regulation of Bax protein expression levels in HCC and leukemia cell lines.

Nordin (26) isolated a coumarin derivative PulchrinA that connected a long alkyl on the coumarin heterocyclic ring from natural product Enicosanthellum pulchrum for the first time and investigated the ability to initiate apoptosis in human ovarian cancer cells. The results showed that Pulchrin A induced the down-regulation of $\mathrm{Bcl}-2$ protein and the up-regulation of Bax protein by activating caspase- 3 and caspase- 9 , inducing strong cytotoxicity to ovarian cancer cells, and the IC50 value was $22 \mu \mathrm{M}$.

Lin (27) synthesized a nitro-coumarin derivate, named 5,7dimethoxy-4-methyl-6-nitro-chromen-2-one, which up-regulated the expression of BAX and PUMA by cleaving PARP, thereby inducing cytotoxicity in wild-type or KRAS mutated colon cancer cells.

Esculetin, a kind of 6,7-dihydroxyl derivative, can be found in various medicinal plants, such as Cichorium intybus, Artemisia capillaries, Ceratostigma willmottianum, Citrus limonia, etc. Arora (28) treated three kinds of pancreatic cancer cell lines with esculetin, and found that the expression of caspase- $3,-8$, and -9 , as well as the activation level and cleavage form in pancreatic cancer cell lines, were significantly increased. Esculetin can also cause the loss of mitochondrial membrane potential in pancreatic cancer cells and increase the cytoplasmic level of cytochrome $\mathrm{C}$, which in turn mediates the activation of caspase- 3 and caspase-9, leading to apoptosis.
Musa (29) synthesized a series of 7,8-Diacetoxy-3-arylcoumarin derivatives (5a-h) and evaluated their cytotoxicity to human prostate (PC-3) and breast cancer (MDA-MB-231) cancer cell lines in vitro. 7,8-Diacetoxy-3-(4-methylsulfonyl phenyl) coumarin (5f) is the most active derivative and has the highest cytotoxicity and selectivity to PC-3 cell lines. $5 \mathrm{f}$ induced the upregulated expression of apoptotic proteins such as caspases 3, caspases8, Bid, and Smac/DIABLO, and also induced PC-3 cells apoptosis by reducing mitochondrial membrane potential.

Han (30) synthesized a series of shikonin derivatives and evaluated their anti-human cervical cancer activity. Among them, PMMB232 showed the best anti-proliferative activity, and the IC50 value was $3.25 \pm 0.35 \mu \mathrm{M}$. PMMB232 induced apoptosis by reducing mitochondrial membrane potential in human cervical cancer cells. PMMB232 also dose-dependently reduced the expression level of PARP and promoted the PARP cleavage.

\section{MDR Inhibition}

MDR refers to the phenomenon of broad-spectrum drug resistance in tumors, an important cause of tumor recurrence. The main mechanism of MDR is that transmembrane proteins such as $\mathrm{p}$ glycoprotein (P-gp) and multidrug resistance-related protein 2 can transfer various anti-tumor drugs from the cytoplasm to the outside of the cells by using the energy from the hydrolysis of ATP. Studies have shown that coumarins can inhibit MDR by inhibiting P-gp or multidrug resistance-related protein 2 .

Baghdadi (31) isolated six kinds of coumarin compounds (mansorin-A, mansorin-B, mansorin-C, mansorins-I, mansorinII, and mansorin-III) from the heartwood of Mansonia gagei family Sterculariaceae, and detected the potential anticancer activities of these compounds against breast cancer, cervical cancer, colorectal cancer, and liver cancer cells. Mansorin-II and mansorin-III showed the best inhibitory effects to these cell lines, with IC50 values ranging from $0.74 \mu \mathrm{M}$ to $36 \mu \mathrm{M}$ and 3.95 to $35.3 \mu \mathrm{M}$, respectively. In addition, mansorin-II can enhance the anticancer effect of taxol. This synergistic effect may be related to interfering with the efflux activity of P-gp pumps.

Based on high affinity inhibitors, by means of molecular docking simulation and pharmacophore study, Tripathi (32) designed and synthesized a series of new coumarin derivatives, which can dock at active site cavity of P-gps, have a higher binding affinity to the target protein P-gp and can more effectively inhibit the efflux process, thereby enhancing the bioavailability of various anti-tumor drugs and reducing the growth of breast cancer stem cells.

Kasaian isolated and purified 14 sesquiterpene coumarins from the roots of 4 species of Feruar plants, and evaluated the MDR reversal properties of these sesquiterpene coumarins in A2780/ RCIS cells (cisplatinum-resistant derivatives of human ovarian cancer cell line A2780P). Some compounds had obvious MDR reversal effects. The combination of nontoxic concentrations of sesquiterpene coumadin $(20 \mu \mathrm{M})$ with cisplatin significantly enhanced the cytotoxicity of cisplatin on A2780/RCIS cells. The results showed that conferdione and samarcand had the highest inhibitory effects on pump efflux of multidrug resistance-related protein 2 (33). 


\section{Inhibition of Microtubule Polymerization}

Microtubules are the main components of the cytoskeleton and play an important role in maintaining cell morphology, cell division and proliferation, and signal material transportation. Clinically, antitumor drugs can inhibit the mitosis of tumor cells by promoting the depolymerization of microtubules or inhibiting their aggregation, so that the tumor cells can be stagnate in the M-phase. There are three drug binding sites on the microtubules, namely colchicine, vincristine, and paclitaxel binding sites. Colchicine, vincristine, and paclitaxel are also commonly used as microtubule inhibitors. In addition, colchicine, vincristine, and paclitaxel are transport substrates for P-gp pumps, so multidrug resistance of tumor cells may occur.

Cao (34) synthesized a series of new 4-substituted coumarins and tested their anti-proliferation ability against a variety of tumor cells. Among them, 5-chloro-n-(2-methoxy-5-(Methyl (2-oxo-2HChromen-4-yl)amino) pentanamide (compound 65) showed strong anti-tumor proliferation ability (IC50 value was 3.5-31.9 $\mathrm{nM})$. Compound 65 disrupted microtubule networks in hepatocellular carcinoma cells in a pattern similar to colchicine, and also induce human ovarian cancer cells to stagnate in the G2/M phase and promote their apoptosis. More importantly, compound 65 showed significant antiproliferative activity to P-gp overexpressed tumor cells, class III $\beta$-tubulin overexpressed tumor cells, and multidrug-resistant tumor cells. Through exploring its mechanism, it was found that compound 65 could target colchicine binding sites, inhibit microtubules polymerization and spindle formation, and was more active than colchicine, and could overcome P-gp pumpmediated multi-drug resistance.

Zhu synthesized a class of 4-substituted coumarin $\mathrm{H} 6$ that has a strong ability to inhibit tumor cell proliferation with the IC50 range between 7 and $47 \mathrm{nM}$, and it also has a significant ability to inhibit tumor growth in paclitaxel-resistant tumor models. The mechanism is related to target colchicine binding site of $\beta$ tubulin. H6 is a powerful microtubule inhibitor. In order to solve its hydrophobicity limitation, Zhu prepared H6/MPEG2kPCL2k micelles by a simple thin-film hydration method, which significantly improve its solubility, reduce its toxicity and extend the half-life of the drug without affecting the anti-cancer property of this derivative (35).

Dahong tested the anti-proliferative activity of Ferulin $\mathrm{C}$ against breast cancer cells in vitro and in vivo. In vitro, the furan coumarin core of Ferulin $\mathrm{C}$ is in contact with $\beta$-tubulin through colchicine binding sites, thereby inhibiting tubulin aggregation. Ferulin $\mathrm{C}$ could markedly suppress the tubulin polymerization with IC50 of $9.2 \mu \mathrm{M}$ and Colchicine (IC50 $=1.8 \mu \mathrm{M}$ ) was used as the reference compound. Further research suggested that Ferulin C only affected the structure of microtubules and had not effect on the expression of tubulin. Ferulin $\mathrm{C}$ induced microtubule instability, and subsequently activated p21 and inhibited PAK1. In breast cancer cells, elevated expression of PAK1 indicated poor survival and p21 was associated with better survival. Ferulin $\mathrm{C}$ induced G1/S cell cycle arrest via p21Cip1/Waf1-CDK2 signaling pathway. Dahong established a breast cancer cells xenograft model and evaluate antitumor activity of Ferulin C (low dose, $25 \mathrm{mg} / \mathrm{kg}$; median dose, $50 \mathrm{mg} / \mathrm{kg}$; high dose, $100 \mathrm{mg} / \mathrm{kg}$ ) in vivo. Ferulin C could significantly inhibit the growth of xenograft breast cancer cells and the antitumor mechanism was significantly consistent with in vitro experiments (21).

\section{Reactive Oxygen Species Regulation}

ROS is a type of natural by-products produced during cell metabolisms, such as superoxide anion, hydrogen peroxide, and peroxide hydrogen root, hydroxyl radical. In normal cells, medium and high concentrations of ROS are believed to be closely related to apoptosis caused by the cellular stress response, while low concentrations of ROS have a wide range of physiological significance, affecting some signaling pathways and activating transcription factors, and promoting cell proliferation and differentiation. In tumor cells, due to excessive metabolic activity and impaired mitochondrial function, ROS levels are much higher. In addition, ROS is required for tumorigenesis, tumor cell survival, proliferation, and metastasis (36). ROS mediates activation of transcription factor $\mathrm{NF}-\kappa \mathrm{B}$, which promotes proliferation and metastasis of tumor cells (37).

The intracellular ROS levels of pancreatic cancer cells treated with or without esculetin were stained with DCFH-DA and detected by flow cytometry. Intracellular ROS and protein levels of the ROSdependent transcription factor NF- $\kappa \mathrm{B}$ decreased with timedependent manner after esculetin exposure (28).

On the other hand, excessive ROS production in cells can induce apoptosis. The ability of ROS to cause severe cell damage and cell death has been used as a way to kill cancer cells. Han evaluated the production of intracellular ROS by DCFH-DA staining. Compared with the control group, the production of ROS in human cervical cancer cells treated with Shikonin derivative PMMB232 was significantly increased (30).

Glutathione is a natural antioxidant that neutralizes harmful ROS produced in normal cells, and its consumption plays a central role in cell death. 7,8-diacetoxy-3-(4-methylsulfonyl phenyl) coumarin (compound 5f) synthesized by Musa can consume glutathione, leading to excessive accumulation of ROS in prostate cancer cells and inducing apoptosis caused by oxidative stress (29).

Esculetin can induce the generation and accumulation of ROS in human throat epidermoid cancer cells, and also block cell cycle in the G1/S phase, thus inducing and promoting cell apoptosis (38).

Copper is found to be elevated in several types of cancer and involved in cancer growth, angiogenesis and metastasis, which has made it a potential target for developing anticancer therapeutics. Saman synthesized a coumarin-based copper chelator, di(2-picolyl) amine-3(bromoacetyl)coumarin hybrid molecule (ligand-L), and tested the anticancer activity of ligand-L in rat model of diethylnitrosamine induced hepatocellular carcinoma, including ex vivo and in vivo experiments. The in vitro suggested that ligand-L $(25 \mu \mathrm{M})$ interacts with cellular copper and generates ROS which causes oxidative DNA damage and cell death of hepatocellular carcinoma cells. In vivo experiments, ligand-L(250 $\mathrm{mg} \mathrm{kg}^{-1}$ body weight) significantly suppressed the progression of diethylnitrosamine -induced liver cancer by producing copper-ligand-L and thereby produces ROS, and showed not toxic to normal rat liver tissue. The in vivo findings revealed that ligand-L shows a chemopreventive 
effect as it decreased levels of serum liver markers and alpha fetoprotein, and improved liver architecture (39).

\section{Inhibition of Tumor Angiogenesis}

Angiogenesis is the process of forming and recruiting new blood vessels from preexisting vascular system, which is necessary for solid tumor growth. Therefore, targeting tumor angiogenesis is one of the most critical anti-tumor strategies.

Naipeng tested the effects of three triphenylethylene-coumarin hybrids (TCHs) on endothelial cell migration and angiogenesis induced by breast cancer cells in vivo and in vitro. Among them, $\mathrm{TCH}-5 \mathrm{C}(5$ and $10 \mu \mathrm{M})$ showed high anti-proliferative activity and low cytotoxicity in human umbilical vein endothelial cells. In addition, TCH-5C $(2.5 \mu \mathrm{M})$ can inhibit the formation of microtubules in human umbilical vein endothelial cells, destroy cytoskeleton, and inhibit the migration of human umbilical vein endothelial cells. TCH-5C (5 $\mu \mathrm{M})$ also increased the expression of cyclin-dependent kinase inhibitor P21 and decreased the level of cyclin B1, which induces cell cycle arrest and inhibits the proliferation of human umbilical vein endothelial cells. In vitro, TCH-5C $(5 \mu \mathrm{M})$ reduced the production and secretion of VEGF in breast cancer cells and directly inhibited the formation and migration of endothelial cell tubes induced by breast cancer cells. In vivo, tumors in the nude mouse model treated with $\mathrm{TCH}-5 \mathrm{C}$ were significantly smaller in size and weight than those in the untreated control group. In the nude mouse model, TCH-5C also reduced serum VEGF levels, suggesting that $\mathrm{TCH}-5 \mathrm{C}$ inhibits tumor progression by reducing VEGF-induced angiogenesis (40).

da Cruz evaluated the toxicity and antitumor mechanism of 7isopentenyloxycoumarin (UMB-07) on Ehrlich ascites carcinoma model. The results showed that the LD50 value of UMB-07 was about $1000 \mathrm{mg} / \mathrm{kg}$, and at the concentration of $50 \mathrm{mg} / \mathrm{kg}$, UMB-07 had significant anti-tumor activity in vivo, indicating that UMB-07 was a good candidate anti-tumor drug worthy of further study. The main antitumor mechanism of UMB-07 is the inhibition of tumor angiogenesis by reducing chemokine CCL2, and CCL2 is important to tumor neovascularization by inducing increased VEGF in tumor microenvironment. However, this coumarin also induced gastrointestinal toxicity and produced a reduction on nutrient absorption, which caused a reduction in the weight of animals and an increase on feed intake (41).

A series of coumarin derivatives incorporating different functional groups were synthesized and tested for their antiproliferative activity against breast cancer and prostate cancer cells. They all revealed more cytotoxic activity on breast cancer cells than the reference standard staurosporine $(\mathrm{IC50}=8.81 \mu \mathrm{M})$ with the IC50 values ranged from 1.24 to $8.68 \mu \mathrm{M}$. Eman further explored the antitumor mechanism of 2-(4-methyl-2-oxo-2Hchromen-7-yloxy)-N'-benzylacetohydrazide(4a), which is the most cytotoxic of all. 4a targeted VEGFR-2 and reduced its activity, thereby inhibiting tumor angiogenesis. 4a also induced preG1 apoptosis and prevented cell growth in G2/M phase and activated caspase-9. However, none of these derivatives showed significant cytotoxic activity on prostate cancer cells (42).

Signal transducer and activator of transcription-3 (STAT3) gene is an oncogene and is overexpressed in many tumor types. STAT3 expression and phosphorylation increased with malignant progression of laryngeal cancer. STAT3 mediates angiogenesis in laryngeal cancer, and inhibition of the JAK-2/Stat-3 signaling pathway significantly inhibits invasion in vitro and angiogenesis of laryngeal cancer. Esculetin inhibits migration and invasion of laryngeal cancer by inhibiting STAT3 phosphorylation and preventing STAT3 transport into the nucleus. In addition, Colivelin can reverse the anticancer effect of Esculetin on laryngeal cancer by activating STAT3, further proving that Esculetin functions by inhibiting STAT3 phosphorylation (38).

\section{Other Mechanisms}

Nrf2, which is strictly regulated by KEAP1, is an important antitumor factor and a major regulator of antioxidant reactions. It can bind to antioxidant reaction elements in the promoters of antioxidant proteins and activate the coding of these proteins (43). In tumor cells, KEAP1 sequesters Nrf2 in the cytoplasm and promotes its ubiquitination and proteasomes degradation (44). In order to detect whether esculetin affects the regulation of the antioxidant reaction elements pathway, Arora treated pancreatic cancer cells with esculetin and detected the interaction between Nrf2 and KEAP1, and found that esculetin could directly bind to KEAP1, then destroying the interaction of Nrf2-KEAP1, releasing Nrf2 from the inhibition of KEAP1, increasing the amount of phosphorylation of Nrf2 in cells and increasing the expression of $\mathrm{NAD}(\mathrm{P}) \mathrm{H}$ quinone oxidoreductase-1 (NQO1), the direct target of Nrf2 (28).

NQO1 is a very important enzyme in human cells, whose functions include xenogenic detoxification, superoxide removal, regulation of p53 proteasome, degradation and maintenance of endogenous antioxidants (45). NQO1 is highly expressed in many tumors and play an important role for tumor growth and migration. Studies have shown that NQO1 may be a target for cancer therapy, and inhibition of NQO1 may improve the efficacy of antitumor chemotherapy. By means of NQO1 activity assay, Khunluck studied the inhibitory effects of 21 natural compounds on NQO1 and found that coumarin compounds (coumarin, aesculetin, umbelliferone, and scopoletin) had the stronger inhibitory effect on NQO1, so these componuds could be used as non-competitive inhibitors of NQO1. The inhibitory rate of scopolamine on NQO1 (77.38\%) was the highest. In cholangiocarcinoma cells, scopoletin and umbelliferone showed a strong inhibitory effect on NQO1, and scopoletin also showed strong cytotoxicity on cholangiocarcinoma cells. In order to explore whether NQO1 was involved in the cytotoxicity of scopoletin to cholangiocarcinoma cells, Khunluck also tested the cytotoxicity of scopoletin to cholangiocarcinoma cells that had been introduced with NQO1 siRNA and found that the cytotoxicity was reduced. Scopoletin can also inhibit the migration of bile duct cancer cells by reducing the mRNA ratio of the migration-related gene MMP9/TIMP1 (46).

Aldehyde dehydrogenase (ALDH), which catalyzes the oxidation of acetaldehyde and other aliphatic aldehydes, is a key enzyme in the ethanol metabolic pathway and an important molecule of antioxidant stress. It is also one of the common markers of normal stem cells and cancer stem cells. In breast cancer cells, highly active ALDH leads to breast cancer stem cell characterization by upregulating Notch-1 and epithelial-mesenchymal markers. 
Activation of the Notch-1 signal plays an important role in the selfrenewal, proliferation, and apoptosis of tumor cells. Abnormal activation of Notch1 is relatively common in many tumor types. Deeksha examined the functional and clinical significance of ALDH expression by immunohistochemical measurements in breast cancer tissue. ALDH expression is significantly higher in higher grade breast tumors tissues (grade II and III) than in normal breast tissue. Injection of breast cancer stem cell (ALDH+ and CD44+/CD22-) cells resulted in invasive tumor growth in athymic mice compared with ALDH- cells. ALDH+ and CD44+/CD22- tumors grow rapidly and are larger than the slow-growing and smaller ALDHtumors. On the molecular level, the ALDH+ tumor has higher expression of Notch-1 and EMT markers than ALDH- tumor. Oral administration of the naturally occurring Psoralidin $(25 \mathrm{mg} / \mathrm{kg}$ of body weight) highly inhibited the growth of ALDH+ and ALDHtumors on xenograft models. In addition, Psoralidin inhibited Notch-1 mediated EMT activation in ALDH+ and ALDHtumors. Therefore, Psoralidin may prevent the incidence and metastasis of breast cancer by significantly inhibiting Notch-1 in breast cancer cells (47).

Monocarboxylate transporter (MCT) transports pyruvate and lactic acid accumulated during glycolysis from tumor cells to avoid cytoplasmic acidification, which maylead to apoptosis. There are 14 known isoforms of MCTs, and MCT1-4 is responsible for transporting these carboxylate. They are also associated with the influx and outflow of lactate, which can produce energy in cancerrelated stromal fibroblasts and epithelial cancer cells. Elevated MCT1 expression has been identified in a large number of cancers, so this transporter may be a target for broad-spectrum cancer therapy. Gurrapu (48) synthesized a series of N, N-dialalkyl carboxyl coumarins as MCT1 inhibitors, and tested cytotoxicity of these coumarins on MCT1 and MCT4 expression cell lines in vitro. The lead compound 4a (7-(dibenzylamino-2-oxo-2h-Chromene3-carboxylic acid) was highly effective in inhibiting glioblastoma that was predominantly MCT1 expression but did not show any activity in triple-negative breast cancer that was MCT4 expression.

HIF-1 is a major activated transcription factor under hypoxia conditions. Its activity is mainly determined by HIF- $1 \alpha$. Members of the HIF- $1 \alpha$ family are key regulators of glycolysis. HIF- $1 \alpha$ is widely overexpressed in human cancers to prevent tumor apoptosis due to oxygen depletion. Activation and stabilization of the HIF- $1 \alpha$ signaling pathway induce the expression of many target genes involved in tumor cell growth, metabolism, and invasion. The shikonin derivative PMMB232 synthesized by Han et al. can bind to HIF- $1 \alpha$, thereby promoting HIF- $1 \alpha$ degradation, impairing glycolysis in cancer cells and promoting apoptosis. PMMB232 can also up-regulate the expression of E-cadherin, enhance cell adhesion, and inhibit the migration and metastasis of tumor cells (30).

AKR1B10 (Aldo-Keto Reductase 1B10), which is highly expressed in normal gastrointestinal epithelial tissues, but low or no expression in other normal tissues, is an NADPH-dependent reductase whose function is mainly to catalyze the reduction of various carbonyl compounds. AKR1B10 has also been found to be overexpressed in human non-small cell carcinoma, liver cancer, breast cancer, oral squamous cell carcinoma and pancreatic cancer, and is involved in the occurrence, development and survival of tumor cells through a variety of mechanisms, including cytotoxic reactive carbonyl compounds Detoxification, enhance the migration and proliferation potential of tumor cells, regulate cellular fatty acid synthesis and lipid metabolism, and participate in the acquisition of multidrug resistance in tumor

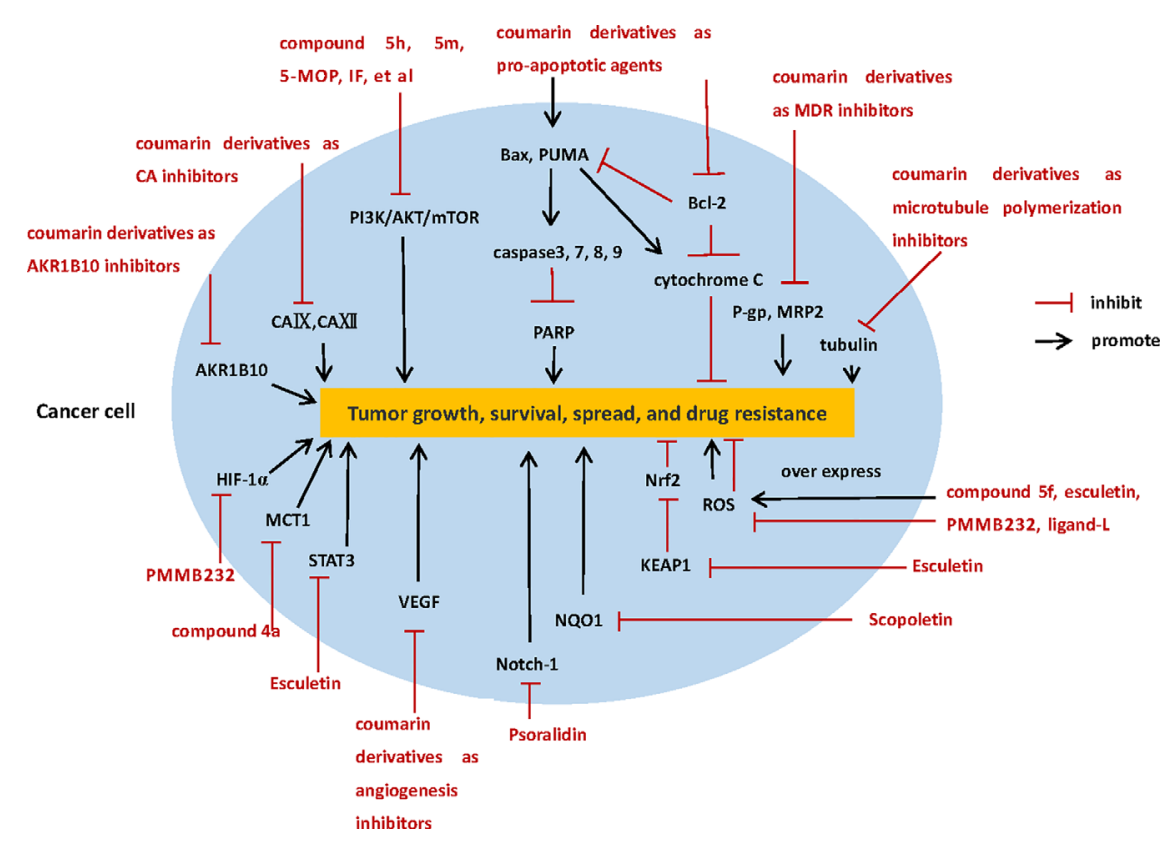

FIGURE 2 | The diverse anti-tumor mechanisms of coumarin derivatives. 
cells. HMCB (7-hydroxy-2-(4-methoxyphenylimino)-2Hchromene-3-carboxylic acid benzylamide) is the most effective AKR1B10 inhibitor currently but does not have a high selectivity. Satoshi et al. took HMCB as a starting material to synthesize a series of coumarin derivatives, which have stronger selectivity and inhibition for AKR1B10. These coumarin derivatives significantly inhibited the migration and proliferation of lung cancer cells, increased the cisplatin sensitivity of cisplatin-resistant lung cancer cells, and inhibited the metastasis and invasion potential of cisplatin-resistant lung cancer cells (49). Awale et al. suggested that AKR1B10 may also be related to the tolerance of pancreatic cancer to nutritional deficiency. Human pancreatic cancer cells are resistant to nutritional starvation by switching energy metabolism, allowing them to survive in tumor microenvironments where blood vessels are scarce. Awale synthesized a series of coumarin derivatives based on HMCB structure and evaluated their inhibition against the resistance to nutritional starvation of human pancreatic cancer cells. 7-hydroxy-2-oxo-2H-chromene3-carboxylic acid (3-phenylpropyl)amide (compound 2c) showed highly selective cytotoxicity on human pancreatic cancer with a

TABLE 1 | Summary of the mechanisms of coumarins as antitumor drugs.

\begin{tabular}{|c|c|c|c|}
\hline Coumarins & Test type & Effective concentration & Mechanisms \\
\hline Compound 8i (5) & & CA IX: 45.5 nM CA XII: 596.6 nM & selective inhibitory activities to CA IX and CA XII \\
\hline Compound $5 p$ and $5 n(6)$ & & CA IX: $144.6 \mathrm{nM}$ and $71.5 \mathrm{nM}$ & \\
\hline 1-aryl and 2-aryl-substituted & & CA IX: $9.6 \mathrm{nM}$ & \\
\hline Coumarin derivatives $(7)$ & & CA XII: 5.8 nM and 25.2 nM & \\
\hline 4-sulfamoylphenyl/sulfocoumarin & In vitro & CA IX: 99.4-4092.5 nM & \\
\hline benzamides (8) & & CA XII: 30.6-7461.5 nM & \\
\hline dihydroartemisinin-coumarin & & CA IX: $0.17 \mu \mathrm{M}$ & \\
\hline hybrids (9) & & CA XII: $0.17 \mu \mathrm{M}$ & \\
\hline $\begin{array}{l}\text { 2-thioxocoumarine derivative } 15 \\
\text { and } 16(10)\end{array}$ & & $0.004-0.027 \mu \mathrm{M}$ & \\
\hline Compound $5 \mathrm{~h}$ and $5 \mathrm{~m}(15)$ & & $18.1-32.6 \mu \mathrm{M}$ and $29.3-42.1 \mu \mathrm{M}$ & inhibition of the expression or phosphorylation of PI3K, Akt, \\
\hline 5-methoxypsoralen (16) & & $75 \mu \mathrm{M}$ & and mTOR \\
\hline Isofraxidin (17) & & $40 \mu \mathrm{M}$ & \\
\hline TCHD (18) & In vitro & $75-125 \mu \mathrm{g} / \mathrm{ml}$ & \\
\hline Imperatorin (19) & & $75 \mu \mathrm{M}$ & \\
\hline $\begin{array}{l}\text { 3-benzyl coumarin deca-B-ring } \\
\text { derivative (20) }\end{array}$ & & $53 \mathrm{nM}$ & \\
\hline Ferulin C (21) & & $20 \mu \mathrm{M}$ & \\
\hline Ferulin C (21) & & $20 \mu \mathrm{M}$ & up-regulate the expression of pro-apoptotic proteins, and \\
\hline $\begin{array}{l}\text { Xanthyoxylin and 6, 7, 8- } \\
\text { trimethoxyl-coumarin (24) }\end{array}$ & & $0.1 \mu \mathrm{g} / \mathrm{ml}$ & down-regulate the expression of anti-apoptotic proteins \\
\hline Isofraxidin (17) & & $10 \mu \mathrm{g} / \mathrm{ml}$ & \\
\hline $\mathrm{TCHD}(18)$ & & $75-125 \mu \mathrm{g} / \mathrm{ml}$ & \\
\hline $\begin{array}{l}\text { 8-methoxy coumarins hybridize } \\
\text { with three bioactive moieties ( } 25)\end{array}$ & In vitro & $0.65-0.93 \mu \mathrm{M}$ & \\
\hline PulchrinA (26) & & $22 \mu \mathrm{M}$ & \\
\hline A nitro-coumarin derivate (27) & & $40 \mu \mathrm{M}$ & \\
\hline Esculetin (28) & & $100 \mu \mathrm{M}$ & up-regulate the expression of pro-apoptotic proteins, and \\
\hline Compound $5 f(29)$ & & $26.43 \mu \mathrm{M}$ & down-regulate the expression of anti-apoptotic proteins; \\
\hline PMMB232 (30) & In vitro & $3.25 \pm 0.35 \mu \mathrm{M}$ & induce the generation and accumulation of ROS overly or \\
\hline Esculetin (38) & In vitro & $10 \mu \mathrm{M}$ & decrease the intracellular ROS level \\
\hline Ligand-L (39) & In vitro & $250 \mathrm{mg} / \mathrm{kg}$ & \\
\hline Mansorin-II; (31) & In vitro & $0.74-36 \mu \mathrm{M}$ & interfer with the efflux activity of P-gp or MRP2 \\
\hline Coumarin derivatives (32) & & & \\
\hline Conferdione and Samarcand (33) & & $22.75 \pm 3.88 \mu \mathrm{M}$ and $>50 \mu \mathrm{M}$ & \\
\hline Ferulin C (21) & In vitro & $25 \mathrm{mg} / \mathrm{kg}$ & target colchicine binding sites, inhibit microtubules \\
\hline Compound 65 (34) & In vitro & $3.5-31.9 \mathrm{nM}$ & polymerization \\
\hline 4-substituted coumarin H6 (35) & & $7-47 \mathrm{nM}$ & \\
\hline $\mathrm{TCH}-5 \mathrm{C}(40)$ & In vitro & $3 \mathrm{mg} / \mathrm{kg}$ & reduce VEGF-induced angiogenesis \\
\hline 7-isopentenyloxycoumarin (41) & In vitro & $50 \mathrm{mg} / \mathrm{kg}$ & \\
\hline Compound $4 \mathrm{a}(42)$ & In vitro & $1.24-8.68 \mu \mathrm{M}$ & \\
\hline Esculetin (38) & In vitro & $10 \mu \mathrm{M}$ & inhibit STAT3 phosphorylation \\
\hline Esculetin (28) & In vitro & $100 \mu \mathrm{M}$ & increase the amount of anti-tumor factor Nrf2 \\
\hline Scopoletin (46) & In vitro & $0.88 \mathrm{mM}$ & inhibitory effect on NQO1 \\
\hline Psoralidin (47) & In vitro & $25 \mathrm{mg} / \mathrm{kg}$ & inhibit Notch-1 in breast cancer cells \\
\hline Compound $4 \mathrm{a}(48)$ & In vitro & $0.09 \pm 0.01 \mu \mathrm{M}$ & inhibition of the expression of MCT1 \\
\hline PMMB232 (30) & In vitro & $4 \mu \mathrm{M}$ & $\begin{array}{l}\text { promote HIF-1 } \alpha \text { degradation; } \\
\text { up-regulate the expression of E-cadherin }\end{array}$ \\
\hline $\begin{array}{l}\text { Coumarin derivatives based on } \\
\text { HMCB (49) }\end{array}$ & In vitro & $4.1 \pm 0.3 \mathrm{nM}$ and $3.4 \pm 0.1 \mathrm{nM}$ & inhibition for AKR1B10 \\
\hline compound 2c (50) & & $0.44 \mathrm{mM}$ & \\
\hline
\end{tabular}


PC50 value of $0.44 \mathrm{mM}$ but no toxicity on conventional nutrientrich media (50).

\section{DISCUSSION}

Coumarin compounds can inhibit the growth, proliferation and metastasis of various tumor cells through a variety of mechanisms, including inhibition of carbonic anhydrase, PI3K/AKT/mTOR signaling pathway, microtubule polymerization, angiogenesis, monocarboxylate transporters, hypoxia-inducible factor-1; acting on apoptosis proteins and inhibiting tumor multidrug resistance, regulation ROS, and so on (Figure 2). Whether it is a natural coumarin extracted from various plants or new coumarin derivative synthesized by modification of the basic structure of coumarin, in vitro experiments, could inhibit the growth and proliferation of tumor cells at low concentration, and did not produce poisonousness to the normal cells, which proved that coumarins are a class of promising anti-tumor drugs with high selectivity.

However, there are still many problems to be solved before coumarins can be used clinically. First, the current studies of coumarins as new antitumor drugs still in a primary stage, many of the studies on the anti-tumor mechanisms of coumarins have not been studied deeply, and stopped at a relatively simple mechanism. Second, most studies are limited in the in vitro experiments, lack of in vivo evidence to support, so the antitumor potential and security of coumarins in vivo is not clear, which needs further research. Third, coumarin compounds are fat-soluble, and have poor solubility in water, in order to enhance their bioavailability, may need to improve the concentration or the use of cosolvent, carrier system to increase their solubility and bioavailability, or through the structure modification or the introduction of new functional groups, can give coumarin compounds new performance. However, these measures may affect the antitumor activity and safety of coumarin compounds in vivo and in vitro.

On the whole, numerous in vitro and in vivo experiments proved coumarins are indeed a kind of potential new antitumor drugs, which have selective cytotoxicity and diverse anti-tumor mechanisms (Table 1), but there are still problems that most of

\section{REFERENCES}

1. Hassanein EHM, Sayed AM, Hussein OE, Mahmoud AM. Coumarins as Modulators of the Keap1/Nrf2/ARE Signaling Pathway. Oxid Med Cell Longev (2020) 2020:1675957. doi: 10.1155/2020/1675957

2. Venugopala KN, Rashmi V, Odhav B. Review on natural coumarin lead compounds for their pharmacological activity. BioMed Res Int (2013) 2013:114. doi: $10.1155 / 2013 / 963248$

3. Neri $\mathrm{D}$, Supuran CT. Interfering with $\mathrm{pH}$ regulation in tumors as a therapeutic strategy. Nat Rev Drug Discovery (2011) 10:767-77. doi: 10.1038/nrd3554

4. Supuran CT. Carbonic anhydrase inhibition and the management of hypoxic tumors. Metabolites (2017) 7(3):48. doi: 10.3390/metabo7030048

5. Kurt BZ, Sonmez F, Ozturk D, Akdemir A, Angeli A, Supuran CT. Synthesis of coumarin-sulfonamide derivatives and determination of their cytotoxicity, carbonic anhydrase inhibitory and molecular docking studies. Eur J Medicinal Chem (2019) 183:111702. doi: 10.1016/j.ejmech.2019.111702

6. Kurt BZ, Dag A, Doğan B, Durdagi S, Angeli A, Nocentini A, et al. Synthesis, biological activity and multiscale molecular modeling studies of bis-coumarins as selective carbonic anhydrase IX and XII inhibitors with effective cytotoxicity these studies are not in-depth or to explore the relationship between mechanisms, lack of in vivo experiments results in its unknown of security and drug metabolism in vivo, and low solubility in water limits its bioavailability, however, we believe that the modifications based on the structure of coumarin can solve these problems in the near future. We still have high hopes for coumarin compounds, because the structure of coumarin determines its many biological pharmacological activities, and the heterocyclic structure of coumarin is easy to bind to a variety of target proteins. The $2 \mathrm{H}$ chromen-2-one ring can interact with different biological antigens because of its aromatic, planar, and lipophilic. In addition, the lactone group of coumarin enables the molecule to form strong polar bonds, such as hydrogen bonds and acetylated protein targets (51). Researchers may be able to link the antitumor mechanisms, water solubility with structure and group, by modifying the structure to produce coumarin derivatives with higher selectivity, less side effects, and higher bioavailability. We look forward to more studies on the anti-tumor mechanisms of coumarins, especially in vivo experiments and preclinical studies, to evaluate the efficacy, safety, and pharmacokinetic properties of coumarins, so as to accelerate the development of coumarins as new anti-tumor drugs and provide a new strategy for clinical cancer treatment.

\section{AUTHOR CONTRIBUTIONS}

YW, JX, and YL wrote the first draft of the manuscript. JX, YL, and $Y Z$ organized the literature search. GW supervised the study and edited the manuscript. YW, JX, YL, and YZ wrote sections of the manuscript. All authors contributed to the article and approved the submitted version.

\section{FUNDING}

This work was supported by the Natural Science Foundation of Hunan Province (Project Number: 2019JJ40365) and the Innovation and Entrepreneurship Training Program for College students of 2020 grade (Project Number: S2020105330352).

against hepatocellular carcinoma. Bioorganic Chem (2019) 87:838-50. doi: 10.1016/j.bioorg.2019.03.003

7. Bonardi A, Falsini M, Catarzi D, Flavia V, Lorenzo DCM, Barbara T, et al. Structural investigations on coumarins leading to chromeno[4,3-c]pyrazol-4ones and pyrano[4,3-c] pyrazol- 4- ones: New scaffolds for the design of the tumor-associated carbonic anhydrase isoforms IX and XII. Eur J Medicinal Chem (2018) 146:47-59. doi: 10.1016/j.ejmech.2018.01.033

8. Angapelly S, Angeli A, Khan AJ, Sri Ramya PV, Supuran Claudiu T, Mohammed A. Synthesis and Biological Evaluation of 4-Sulfamoylphenyl/ Sulfocoumarin Carboxamides as Selective Inhibitors of Carbonic Anhydrase Isoforms hCA II, IX, and XII. ChemMedChem (2018) 13(12):1165-71. doi: $10.1002 / \mathrm{cmdc} .201800180$

9. Yu H, Hou Z, Tian Y, Mou Y, Guo C. Design, synthesis, cytotoxicity and mechanism of novel dihydroartemisinin-coumarin hybrids as potential anticancer agents. Eur J Med Chem (2018) 151:434-49. doi: 10.1016/j.ejmech. 2018.04.005

10. Ferraroni M, Carta F, Scozzafava A, Supuran Claudiu T. Thioxocoumarins Show an Alternative Carbonic Anhydrase Inhibition Mechanism Compared to Coumarins. J Medicinal Chem (2016) 59(1):462-73. doi: 10.1021/acs.jmedchem.5b01720 
11. Yu JS, Cui W. Proliferation, survival and metabolism: the role of PI3K/AKT/ mTOR signalling in pluripotency and cell fate determination. Development (2016) 143(17):3050-60. doi: 10.1242/dev.137075

12. Wee P, Wang Z. Epidermal Growth Factor Receptor Cell Proliferation Signaling Pathways. Cancers (Basel) (2017) 9(12):52. doi: 10.3390/cancers 9050052

13. Martini M, De Santis MC, Braccini L, Gulluni F, Hirsch E. PI3K/AKT signaling pathway and cancer: An updated review. Ann Med (2014) 46 (6):372-83. doi: 10.3109/07853890.2014.912836

14. Mayer IA, Arteaga CL. The PI3K/AKT Pathway as a Target for Cancer Treatment. Annu Rev Med (2016) 67(1):11-28. doi: 10.1146/annurev-med062913-051343

15. Wang T, Peng T, Wen X, Wang G, Sun YB, Liu SC, et al. Design, Synthesis and Preliminary Biological Evaluation of Benzylsulfone Coumarin Derivatives as Anti-Cancer Agents. Molecules (Basel Switzerland) (2019) 24(22):4034. doi: 10.3390/molecules24224034

16. Guo H, He Y, Bu C, Peng ZY. Antitumor and apoptotic effects of 5methoxypsoralen in U87MG human glioma cells and its effect on cell cycle, autophagy and PI3K/Akt signaling pathway. Arch Med Sci AMS (2019) 15 (6):1530-8. doi: 10.5114/aoms.2019.81729

17. Shen P, Wang HG, Li MM, Ma QY, Zhou CW, Pan F, et al. Isofraxidin inhibited proliferation and induced apoptosis via blockage of Akt pathway in human colorectal cancer cells. Biomed Pharmacother (2017) 92:78-85. doi: 10.1016/j.biopha.2017.05.065

18. Jiang J, Wang B, Li J, Ye BD, Lin SY, Qian WB, et al. Total coumarins of Hedyotis diffusa induces apoptosis of myelodysplastic syndrome SKM-1 cells by activation of caspases and inhibition of PI3K/Akt pathway proteins. J Ethnopharmacol (2017) 196:253-60. doi: 10.1016/j.jep.2016.12.012

19. Am JU, Gong WJ, Su Y, Mou ZB. Imperatorin shows selective antitumor effects in SGC-7901 human gastric adenocarcinoma cells by inducing apoptosis, cell cycle arrest and targeting PI3K/Akt/m-TOR signalling pathway. J BUON (2017) 22(6):1471-6.

20. Dong MX, Ye T, Bi YY, Wang Q, Kuerban KDLD, Li JY, et al. A novel hybrid of 3-benzyl coumarin seco-B-ring derivative and phenylsulfonylfuroxan induces apoptosis and autophagy in non-small-cell lung cancer. Phytomedicine (2019) 52:79-88. doi: 10.1016/j.phymed.2018.09.216

21. Yao D, Pan D, Zhen Y, Huang J, Wang J, Zhang J, et al. Ferulin C triggers potent PAK1 and p21-mediated anti-tumor effects in breast cancer by inhibiting Tubulin polymerization in vitro and in vivo. Pharmacol Res (2020) 2020:152. doi: 10.1016/j.phrs.2019.104605

22. Kumar S. Caspase function in programmed cell death. Cell Death Differentiation (2007) 14(1):32-43. doi: 10.1038/sj.cdd.4402060

23. Ivana Scovassi A, Diederich M. Modulation of poly(ADP-ribosylation) in apoptotic cells. Biochem Pharmacol (2004) 68(6):1041-7. doi: 10.1016/j.bcp. 2004.04 .023

24. Yao GD, Cheng ZY, Shang XY, Gao PY, Huang XX, Song SJ. Coumarins from the bark of Juglans mandshurica exhibited anti-hepatoma activities via inducing apoptosis. J Asian Natural Products Res (2017) 19(11):1134-42. doi: 10.1080/10286020.2017.1292256

25. Elshemy HAH, Zaki MA. Design and synthesis of new coumarin hybrids and insight into their mode of antiproliferative action. Bioorganic Medicinal Chem (2017) 25(3):1066-75. doi: 10.1016/j.bmc.2016.12.019

26. Nordin N, Fadaeinasab M, Mohan S, Hashim NM, Othman R, Karimian H, et al. a New Natural Coumarin Derivative of Enicosanthellum pulchrum, Induces Apoptosis in Ovarian Cancer Cells via Intrinsic Pathway. PLoS One (2016) 11(5):e0154023. doi: 10.1371/journal.pone.0154023

27. Lin MH, Wang JS, Hsieh YC, Zheng JH, Cho EC. NO2 functionalized coumarin derivatives suppress cancer progression and facilitate apoptotic cell death in KRAS mutant colon cancer. Chemico Biol Interact (2019) 309:108708. doi: 10.1016/j.cbi.2019.06.021

28. Arora R, Sawney S, Saini V, Steffi C, Tiwari M, Saluja D. Esculetin induces antiproliferative and apoptotic response in pancreatic cancer cells by directly binding to KEAP1. Mol Cancer (2016) 15(1):64. doi: 10.1186/s12943-016-0550-2

29. Musa MA, Latinwo LM, Joseph MY, Badisa Veera L. Identification of 7,8Diacetoxy-3-Arylcoumarin Derivative as a Selective Cytotoxic and Apoptosisinducing Agent in a Human Prostate Cancer Cell Line. Anticancer Res (2017) 37(11):6005-14. doi: 10.21873/anticanres.12047

30. Han HW, Zheng CS, Chu SJ, Sun WX, Han LJ, Yang RW, et al. The evaluation of potent antitumor activities of shikonin coumarin-carboxylic acid,
PMMB232 through HIF-1 $\alpha$-mediated apoptosis. Biomed Pharmacother (2017) 97:656-66. doi: 10.1016/j.biopha.2017.10.159

31. Baghdadi MA, Al-Abbasi FA, El-Halawany AM, Aseeri A, Al-Abd A. Anticancer Profiling for Coumarins and Related O-Naphthoquinones from Mansonia gagei against Solid Tumor Cells In Vitro. Molecules (Basel Switzerland) (2018) 23(5):1020. doi: 10.3390/molecules 23051020

32. Tripathi A, Misra K. Inhibition of P-Glycoprotein Mediated Efflux of Paclitaxel by Coumarin Derivatives in Cancer Stem Cells: An In Silico Approach. Combinatorial Chem High Throughput Screen (2016) 19(6):497506. doi: 10.2174/1386207319666160517115158

33. Kasaian J, Mosaffa F, Behravan J, Masullo M, Piacente S, Iranshahi M. Modulation of Multidrug Resistance Protein 2 Efflux in the Cisplatin Resistance Human Ovarian Carcinoma Cells A2780/RCIS by Sesquiterpene Coumarins. Phytother Res (2016) 30(1):84-9. doi: 10.1002/ptr.5504

34. Cao D, Liu YB, Yan W, Wang CY, Bai P, Wang TJ, et al. Design, Synthesis, and Evaluation of in Vitro and in Vivo Anticancer Activity of 4-Substituted Coumarins: A Novel Class of Potent Tubulin Polymerization Inhibitors. J Medicinal Chem (2016) 59(12):5721-39. doi: 10.1021/acs.jmedchem.6b00158

35. Zhu ZJ, Su ZY, Yang JH, Liu HL, Tang MH, Hu QQ, et al. Anti-Tumor Study of H6, a 4-Substituted Coumarins Derivative, Loaded Biodegradable SelfAssembly Nano-Micelles In Vitro and In Vivo. J Biomed Nanotechnol (2019) 15(7):1515-31. doi: 10.1166/jbn.2019.2797

36. Gupta SC, Hevia D, Patchva S, Park B, Koh W, Aggarwal BB. Upsides and downsides of reactive oxygen species for cancer: the roles of reactive oxygen species in tumorigenesis, prevention, and therapy. Antioxid Redox Signal (2012) 16:1295-322. doi: 10.1089/ars.2011.4414

37. Shishodia S, Amin HM, Lai R, Aggarwal BB. Curcumin (diferuloylmethane) inhibits constitutive NF-kappaB activation, induces G1/S arrest, suppresses proliferation, and induces apoptosis in mantle cell lymphoma. Biochem Pharmacol (2005) 70:700-13. doi: 10.21873/anticanres.12047

38. Zhang G, Xu Y, Zhou HF. Esculetin Inhibits Proliferation, Invasion, and Migration of Laryngeal Cancer In Vitro and In Vivo by Inhibiting Janus Kinas (JAK)-Signal Transducer and Activator of Transcription-3 (STAT3) Activation. Med Sci Monit (2019) 25:7853-63. doi: 10.12659/msm.916246

39. Khan S, Zafar A, Naseem I. Redox cycling of copper by coumarin-di(2-picolyl) amine hybrid molecule leads to ROS-mediated modulation of redox scavengers, DNA damage and cell death in diethylnitrosamine induced hepatocellular carcinoma. Bioorganic Chem (2020) 99:103818. doi: 10.1016/ j.bioorg.2020.103818

40. Cui N, Lin DD, Shen Y, Shi J-G, Wang B, Zhao M-Z, et al. TriphenylethyleneCoumarin Hybrid TCH-5c Suppresses Tumorigenic Progression in Breast Cancer Mainly Through Its Inhibition of Angiogenesis. Anti Cancer Agents Medicinal Chem (2019) 19(10):1253-61. doi: 10.2174/1871520619666190404155230

41. da Cruz RMD, Batista TM, de Sousa TKG, Mangueira VM, Dos Santos JAF, de Abrantes RA, et al. Coumarin derivative 7-isopentenyloxycoumarin induces in vivo antitumor activity by inhibit angiogenesis via CCL2 chemokine decrease. Naunyn-Schmiedeberg's Arch Pharmacol (2020) 2020:1701-14. doi: 10.1007/s00210-020-01884-4

42. Ahmed EY, Abdel Latif NA, El-Mansy MF, Elserwy WS, Abdelhafez OM. VEGFR-2 inhibiting effect and molecular modeling of newly synthesized coumarin derivatives as anti-breast cancer agents. Bioorganic Medicinal Chem (2020) 28(5):115328. doi: 10.1016/j.bmc.2020.115328

43. Bocci V, Valacchi G. Nrf2 activation as target to implement therapeutic treatments. Front Chem (2015) 3:4. doi: 10.3389/fchem.2015.00004

44. McMahon M, Itoh K, Yamamoto M, Hayes JD. Keap1-dependent proteasomal degradation of transcription factor Nrf2 contributes to the negative regulation of antioxidant response element-driven gene expression. J Biol Chem (2003) 278(24):21592-600. doi: 10.1074/jbc.m300931200

45. Siegel D, Gustafson DL, Dehn DL, Han JY, Boonchoong P, Berliner LJ, et al. $\mathrm{NAD}(\mathrm{P}) \mathrm{H}$ : quinone oxidoreductase 1: role as a superoxide scavenger. $\mathrm{Mol}$ Pharmacol (2004) 65(5):1238-47. doi: 10.1124/mol.65.5.1238

46. Khunluck T, Kukongviriyapan V, Senggunprai L, Duangarsong W, Prawan A. The Inhibition Kinetics and Potential Anti-Migration Activity of NQO1 Inhibitory Coumarins on Cholangiocarcinoma Cells. Integr Cancer Therapies (2018) 18:153473541882044. doi: 10.1177/1534735418820444

47. Pal D, Kolluru V, Chandrasekaran B, Baby BV, Aman M, Suman S, et al. Targeting aberrant expression of Notch- 1 in ALDH+ cancer stem cells in breast cancer. Mol Carcinogenesis (2017) 56(3):1127-36. doi: 10.1002/mc.22579 
48. Gurrapu S, Jonnalagadda SK, Alam MA, Ronayne CT, Nelson GL, Solano LN, et al. Coumarin carboxylic acids as monocarboxylate transporter 1 inhibitors: In vitro and in vivo studies as potential anticancer agents. Bioorganic Medicinal Chem Lett (2016) 26(14):3282-6. doi: 10.1016/j.bmcl.2016.05.054

49. Endo S, Xia S, Suyama M, Morikawa Y, Oguri H, Hu D, et al. Synthesis of potent and selective inhibitors of aldo-keto reductase 1B10 and their efficacy against proliferation, metastasis, and cisplatin resistance of lung cancer cells. J Medicinal Chem (2017) 60(20):8441-55. doi: 10.1021/acs.jmedchem.7b0 0830

50. Awale S, Okada T, Dibwe DF, Maruyama T, Takahara S, Okada T, et al. Design and synthesis of functionalized coumarins as potential anti-austerity agents that eliminates cancer cells' tolerance to nutrition starvation. Bioorganic Medicinal Chem Lett (2019) 29(14):1779-84. doi: 10.1016/j.bmc 1.2019.05.010
51. Stefanachi A, Leonetti F, Pisani L, Catto M, Carotti A. Coumarin: A Natural, Privileged and Versatile Scaffold for Bioactive Compounds. Molecules (2018) 23:250. doi: $10.3390 /$ molecules 23020250

Conflict of Interest: The authors declare that the research was conducted in the absence of any commercial or financial relationships that could be construed as a potential conflict of interest.

Copyright (c) $2020 \mathrm{Wu}, \mathrm{Xu}$, Liu, Zeng and Wu. This is an open-access article distributed under the terms of the Creative Commons Attribution License (CC BY). The use, distribution or reproduction in other forums is permitted, provided the original author(s) and the copyright owner(s) are credited and that the original publication in this journal is cited, in accordance with accepted academic practice. No use, distribution or reproduction is permitted which does not comply with these terms. 